\title{
Toward Smart Control of Turbulent Jet Mixing and Combustion*
}

\author{
Nobuhide KASAGI**
}

\begin{abstract}
Recent progress in active control of jet mixing and combustion is introduced. Miniature electromagnetic flap actuators are mounted on the periphery of an axisymmetric nozzle exit. It is demonstrated that even weak disturbances introduced into the initial shear layer by these actuators can significantly modify the large-scale vortical structures. This control technique is extended to the control of methane/air mixing and diffusion combustion by using a coaxial jet nozzle with the same flap actuators. As a result, the flame characteristics can be much improved in terms of stability and emission. Direct numerical simulation of a confined coaxial jet control has also been carried out. Although the distributed actuators are modeled somewhat ideally, DNS clearly demonstrates enormous effects of the present control scheme on the initial shear layer dynamics and concentration mixing.
\end{abstract}

Key Words: Jet, Flow Control, Vortex, Turbulent Mixing, Combustion, Flame, Laser, Measurement

\section{Introduction}

The modern turbulence research has a history of more than 100 years since the Osborne Reynolds' pioneering work in the late 19th century. Its three major aims have been to understand highly nonlinear turbulence mechanics, develop predictive methods for turbulent flow phenomena and devise schemes of controlling them. It is this third target that we currently aim at, and our efforts are now directed toward innovative highly-advanced control methodology, namely, "smart control of turbulence" (Kasagi et al. ${ }^{(1)}$ ), for the enrichment of future human life.

Control of turbulent flows and associated transport phenomena plays a key role in many engineering applications and will be of growing importance in resolving global environmental problems. Its potential impacts can be easily recognized if one thinks about the significance of the artificial manipulation of turbulent drag, noise, heat transfer as well as chemical reaction, to name a few. This important research target has been focused upon over the several decades, and now there is a considerable degree of knowledge on such conventional methods as roughness/grooves, twisted tapes, blowing/suction, sound/vibration, and polymer additives. Unlike these, smart control of turbulence denotes sophisticated interactive control schemes, particularly those exploiting emerg-

\footnotetext{
* Received 6th February, 2006 (No. 06-4055)
}

** Department of Mechanical Engineering, The University of Tokyo, 7-3-1 Hongo, Bunkyo-ku, Tokyo 113-8656, Japan. E-mail: kasagi@ thtlab.t.u-tokyo.ac.jp ing micromachining technology (Ho \& $\left.\mathrm{Tai}^{(2)}\right)$. The progress in this direction is even more accelerated by the development of new research tools such as large-scale numerical simulation and digital imaging techniques applied to detailed study of turbulent flow mechanism.

Among typical canonical turbulent shear flows, single and coaxial jets are of great importance for a variety of applications such as aero and automobile engines, chemical plants, iron and glass manufacturing, water-jet machining, electronic equipment cooling, printing and drying. Hence, there have been many studies on the jet flow dynamics and its control. In this flow mode, the initial shear layer is extremely sensitive to small disturbances, which are amplified through their inherent instability (e.g., Huerre \& Monkewitz $\left.{ }^{(3)}\right)$. As a result, large-scale vortical structures emerge at the frequency of disturbance.

Bulk forcing of a jet column was often used to alter the vortex shedding, and much work has been carried out to investigate the mixing and entrainment properties (Winant \& Browand $^{(4)}$; Crow \& Champagne ${ }^{(5)}$; Zaman \& Hussain ${ }^{(6)}$; Gutmark \& $\mathrm{Ho}^{(7)}$; Mankbadi ${ }^{(8)}$ ). More recently, non-axisymmetric control methods were also attempted to achieve large alterations. Zaman et al. ${ }^{(9)}$ and Reeder \& Samimy ${ }^{(10)}$ examined the effect of tab vortex generators placed inside the exit of a round jet nozzle. They found that each tab produced a pair of counterrotating streamwise vortices, and that the entrainment of the ambient fluid was significantly enhanced with a relatively small thrust penalty.

Active control strategies have also been pursued in 
order to achieve more drastic and flexible flow modifications. Corke \& Kusek $^{(11)}$ introduced localized acoustic disturbances, which were produced by an array of miniature loud speakers placed around the jet nozzle exit. Smith $\&$ Glezer ${ }^{(12)}$ successfully applied miniature zero-massflux jet actuators (synthetic jets) for the control of a largescale planar jet. They showed that the primary jet could be vectored either toward or away from the synthetic jet actuators by creating low-pressure recirculating flow regions. Lee \& Reynolds ${ }^{(13)}$ and Parekh et al. ${ }^{(14)}$ showed that it is possible to make a round jet bifurcate by imposing orbital and axial modes of forcing simultaneously. The requisite condition for the bifurcation is an orbital forcing frequency equal to twice the axial forcing frequency. Danaila \& Boersma ${ }^{(15)}$ investigated the mechanism of a bifurcating jet with the aid of direct numerical simulation. They imposed an oscillating component, which consists of a pair of counter-rotating helical modes, on the nozzle exit velocity, and showed that the single-frequency excitation could trigger a bifurcating jet.

The fact that the small disturbance at the initial boundary layer can alter the entire jet flow field stimulated us to pursue a possibility of producing various jet flow modes with a single nozzle, utilizing distributed MEMSbased actuators, which can be driven independently according to a driving signal supplied by a control program. In most of the previous studies mentioned above, the control input mode introduced is limited to one or a few. Corke and Kusek ${ }^{(11)}$ studied the effect of the jet nozzle equipped with 12 miniature speakers mounted at the exit. Their control modes were only the helical modes of $n=0$ and \pm 1 , where $n$ denotes the azimuthal wave number.

In addition to the single jet control, coaxial jet control is also important for mixing and combustion. An example is a fuel jet surrounded by an annular air coflow, which forms a diffusion flame. If the velocity of fuel and/or air flow is larger than a critical value but smaller than the blowoff limit, the flame is lifted at a certain downstream distance from the nozzle exit. In designing industrial burners, it is of practical importance to predict the liftoff height and the liftoff/blowoff conditions, which many studies have focused on (Pitts $\left.{ }^{(16)}\right)$.

Vanquickenborne \& Triggelen ${ }^{(17)}$ derived an empirical relation between the turbulent burning velocity and characteristics of turbulence. Peters \& Williams ${ }^{(18)}$ concluded that the liftoff process is controlled by the quenching of laminar diffusion flamelets, which is determined by a local scalar dissipation rate. Recently, Vervisch ${ }^{(19)}$ considered that fuel and air are partially premixed upstream of the flame base and that a laminar triple flame plays a dominant role in the flame stabilization mechanism. Muniz $\&$ Mungal $^{(20)}$ found that the lifted flame blows off when the velocity of the surrounding air flow is more than three times larger than the laminar burning velocity.
Fujimori et al. ${ }^{(21)}$ showed that NOx emission is reduced significantly if the liftoff height is larger than the attached flame length, because lean mixture is supplied to the flame through complete mixing upstream. Chao et al. ${ }^{(22)}$ examined NOx and unburnt hydrocarbons (UHC) emissions in the propane/air lifted flame excited with an acoustic speaker. They reported that mixing between the premixture and ambient air is enhanced upstream of the flame base, so that NOx emission is reduced without significant increase of UHC.

With the background above, we have been studying the control strategy utilizing distributed miniature actuators, which has several advantages over the conventional bulk forcing scheme. First, the mechanical disturbance can be introduced into the initial jet shear layer locally and directly, so that the large-scale vortical structures can be produced with a smaller power input. Second, the control mode and frequency can be suitably selected and easily changed depending on the flow condition and the control target. Thus, it can be applied to various jet flows in wide engineering applications, where the flow conditions may change time-dependently.

For this purpose, miniature electromagnetic flap actuators are fabricated and mounted on the periphery of a round jet nozzle exit, which is named as "intelligent nozzle" (Suzuki et al. $\left.{ }^{(23)}\right)$. This nozzle has also been used as a coaxial jet mixer and a diffusion combustor (Kurimoto et al. ${ }^{(24),(25)}$ ). In the latter, a lifted diffusion flame is controlled for the sake of combustion stability and reduced toxic emissions.

\section{Single Jet Flow Control}

Figure 1 shows the electromagnetic flap actuator $(9 \times$ $3 \mathrm{~mm}^{2}$ ) made of a copper plated polyimide film (Suzuki et al. $\left.{ }^{(23)}\right)$. The polyimide and copper layer thicknesses are 25 and $35 \mu \mathrm{m}$, respectively. A square copper coil is patterned lithographically and etched by ferric chloride solution. When the electric current is applied to the coil, the flap is elastically bent by the electromagnetic force between the coil and a cylindrical permanent magnet of $4000 \mathrm{G}$ placed underneath. The displacement of the flap is about $0.3 \mathrm{~mm}$ with $0.8 \mathrm{~A}$ up to $10 \mathrm{~Hz}$ in both air and water, and gradually increases to $0.8 \mathrm{~mm}$ at the first resonant frequency of about $300 \mathrm{~Hz}$ in air and $0.5 \mathrm{~mm}$ at $60 \mathrm{~Hz}$ in water, respectively. In the following, the driving frequency, which is set to around the preferred mode frequency of the jet, stays less than $10 \mathrm{~Hz}$. Therefore, a good flap response to even a square wave signal is accomplished with negligible time delay. Note that flow visualization is made in a recirculating water tank with the jet centerline exit velocity of $U_{\mathrm{o}}=150 \mathrm{~mm} / \mathrm{s}$, which corresponds to $R e_{D}=3000$, unless otherwise noted. The amplitude of the flap actuation is kept at $0.3 \mathrm{~mm}(0.015 \mathrm{D})$ by adjusting the driving current. 

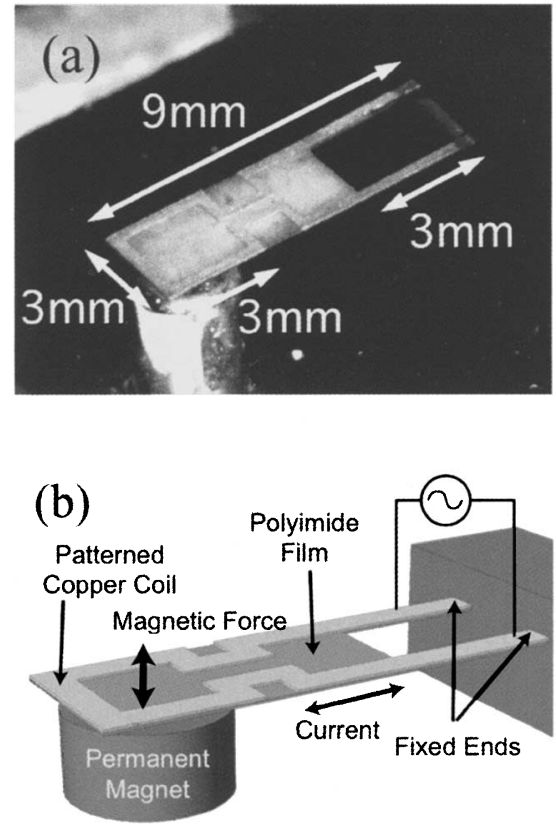

Fig. 1 Overview of an electromagnetic flap actuator and its driving mechanism

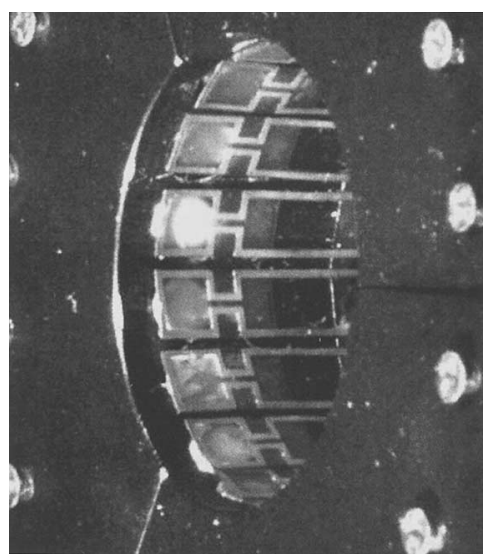

Fig. 2 Jet nozzle equipped with eighteen flap actuators

Figure 2 shows a perspective view of the intelligent nozzle (diameter: $D=20 \mathrm{~mm}$ ) equipped with eighteen electromagnetic flap actuators inside the nozzle exit. Cylindrical permanent magnets are embedded in the nozzle inside wall. These flaps are placed at regular intervals and cover $86 \%$ of the circumference. The neutral position of the flaps is in contact with the magnet, so that they can move only upward. Each flap is driven independently by the amplified electric current, which a multi-channel digital-analog board generates.

A typical longitudinal sectional view of a natural jet is shown in Fig. 3. A laminar shear layer separates from the nozzle lip and rolls up into vortex rings at about $z / D=2.5$. After experiencing some stages of coalescence, ring structures break down into three-dimensional turbulent puffs at $z / D \sim 4$. Figure 3 (b) shows the jet controlled by an axisymmetric flap motion, in which all eighteen flaps are
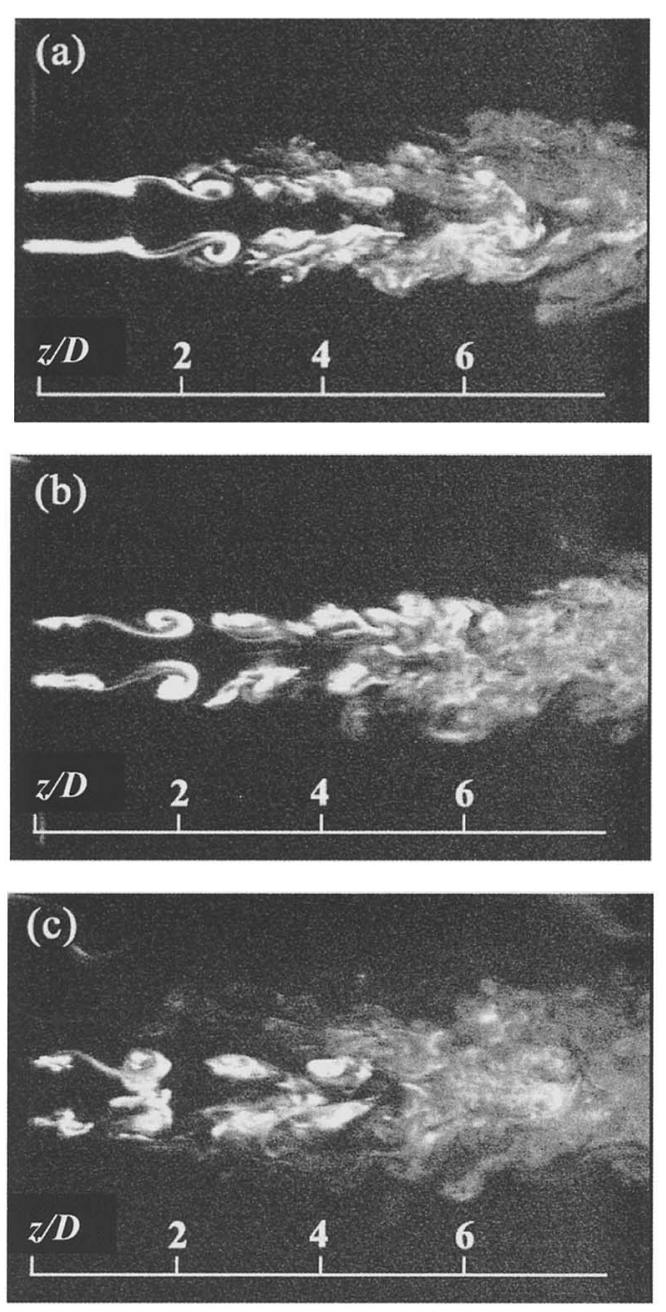

Fig. 3 Longitudinal sectional views of the jet shear layer: (a) natural jet, (b) axisymmetric mode with sinusoidal wave signal at $S t_{\mathrm{a}}=0.5$, (c) axisymmetric mode with square wave signal at $S t_{\mathrm{a}}=0.5$

driven in phase by a sinusoidal wave signal of $f_{\mathrm{a}}=4 \mathrm{~Hz}$ $\left(S t_{\mathrm{a}}=f_{\mathrm{a}} D / U_{\mathrm{o}} \sim 0.5\right)$. The discrete axisymmetric vortex rings roll up at $z / D \sim 2$ with regular spacing, being synchronized with the flap motion. Figure 3 (c) shows the jet excited with a square wave signal at the corresponding phase. In this case, the location of vortex roll-up moves upstream to $z / D \sim 1.5$. It is confirmed repeatedly that the vortex rings start to roll up closer to the nozzle exit with the square wave signal than the sinusoidal wave signal. Hereafter, axisymmetric mode refers an axisymmetric flap motion with a square wave signal.

Among various control modes examined, the most significant effect is observed when upper and lower halves of the eighteen flaps are driven 180 degrees out of phase (alternate mode; hereafter referred to as AL); i.e., the flaps numbered $i=1 \sim 9$ and $10 \sim 18$ are driven by out-of-phase square signals (Fig. 4). Figure 5 (a) shows a longitudinal sectional view of the AL-controlled jet at $f_{\mathrm{a}}=1.9 \mathrm{~Hz}$ $\left(S t_{\mathrm{a}}=0.25\right)$. In this case, the jet clearly bifurcates into two separate jets from $z / D=3$ (see, also a close-up view in 


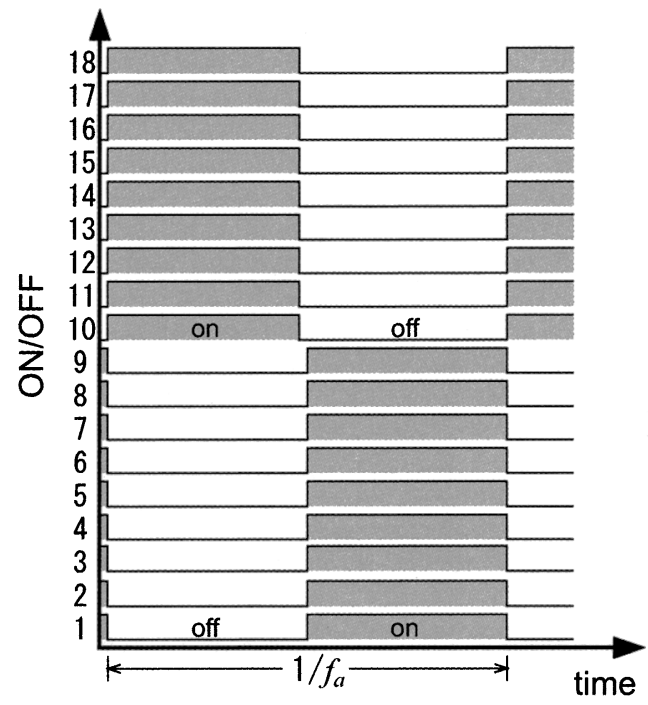

Fig. 4 Control signal diagram of alternate mode
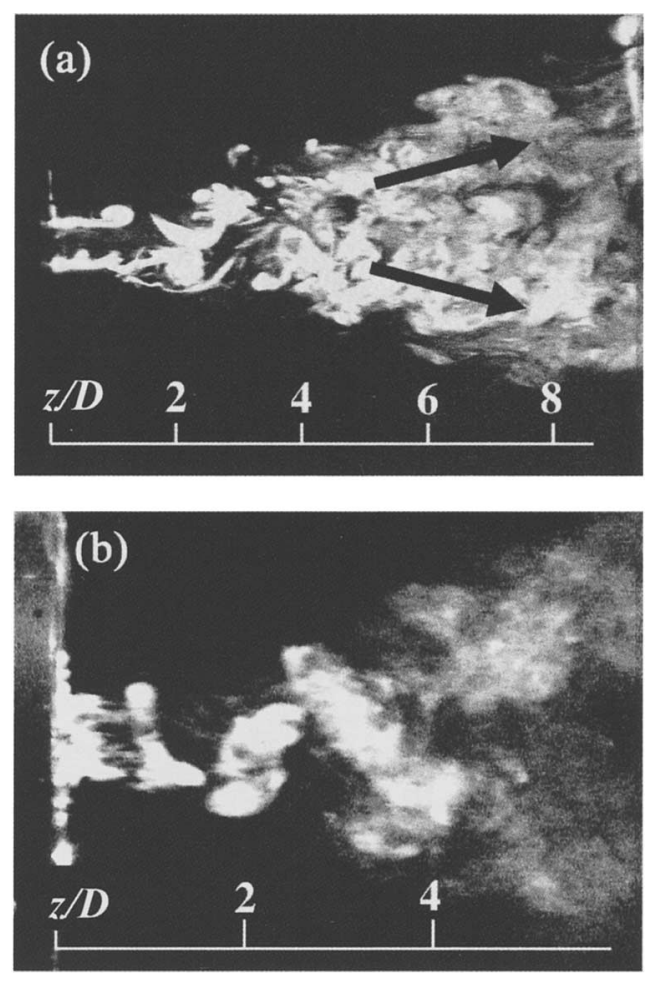

Fig. 5 Bifurcating jet excited with alternate mode at $S t_{\mathrm{a}}=0.25$; (a) longitudinal sectional view, (b) close-up view

Fig. 5 (b)). Alternately inclined and bent vortex rings are shed at a frequency of $2 f_{\mathrm{a}}$. Therefore, two vortices are shed during one cycle of the flap motion. The marked difference from axisymmetric mode is that one half of the vortex is generated by the flap ascending (toward the jet centerline), while the other half is generated by the flap descending (toward the nozzle inside wall). Thus, the vortex rings generated by the AL control have non-uniform vorticity distribution along its circumference. As the vortices are convected downstream, they are transported into

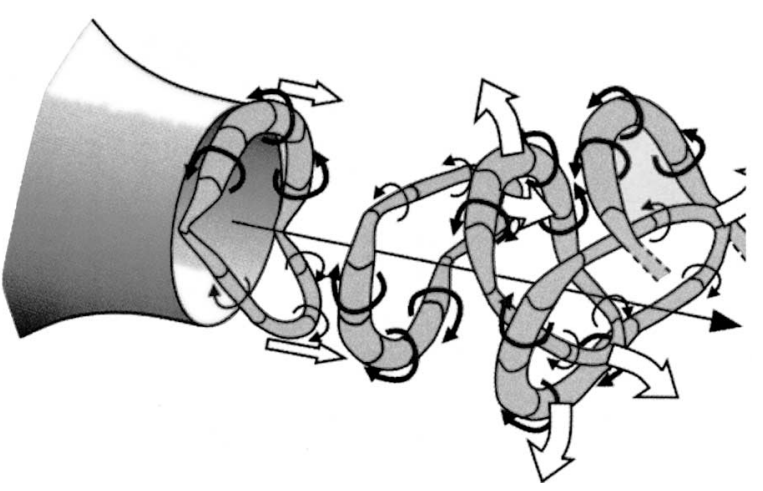

Fig. 6 Proposed model of vortex structures and their motion in the bifurcating jet generated with alternate mode modulation

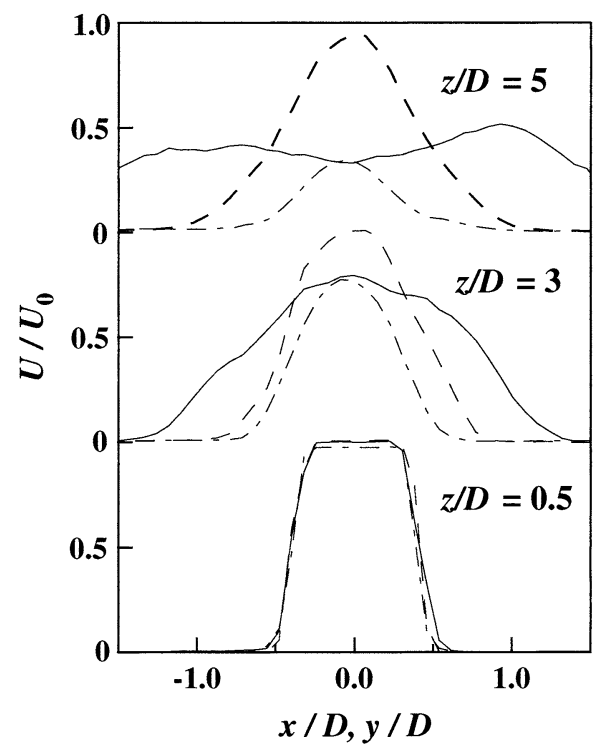

Fig. 7 Cross-stream distributions of streamwise mean velocity. Natural jet: - - - - - Bifurcating jet: bifurcating plane, --_-_-_-- bisecting plane

two different directions from $z / D \sim 3$ by their mutual interaction.

A possible scenario of the bifurcation mechanism is schematically given in Fig. 6. With AL control, a vortex ring with a different magnitude of vorticity between upper and lower halves rolls up; the stronger half is generated by the flap descending, and the weaker half is generated by the flap ascending. As the weaker half is convected faster than the stronger half and approaches the stronger half of the preceding vortex ring, it overtakes the preceding vortex ring. Because of their mutual interaction, both halves are directed toward the off-axis directions. Consequently, the vortex rings are strongly inclined, and induce the alternate off-axis velocity onto the jet core fluid.

Figure 7 shows the radial distributions of the streamwise mean velocity $U$ at various axial locations. The velocity profiles near the nozzle exit $(z / D=0.5)$ are almost identical in all cases. At $z / D=3$, where the bifurcation 
takes place, the centerline velocity in the bifurcating jet is smaller than that in the natural jet. The profile becomes wider in the bifurcating plane, while it is narrower in the bisecting plane. At $z / D=5$, the profile in the bifurcating plane clearly exhibits two separate peaks and a trough in between.

\section{Control of Coaxial Jet Flow and Combustion}

Axisymmetric coaxial jet nozzle is assembled as shown in Fig. 8 (Kurimoto et al. ${ }^{(24),(25)}$ ). The central jet is issued from an installed straight tube of an inner diameter of $D_{\mathrm{i}}=10 \mathrm{~mm}$ and a wall thickness of $0.3 \mathrm{~mm}$. The resultant diameter ratio $\beta\left(=D_{\mathrm{o}} / D_{\mathrm{i}}\right)$ is 2 . The power consumption is 0.2 and $0.5 \mathrm{~W}$ for the saw- and square-wave signals, respectively.

Schematic of the experimental setup is shown in Fig. 9. The central and annular flows are methane and air, respectively. The filtered air is introduced into a plenum chamber with a honeycomb and several meshes. Flow

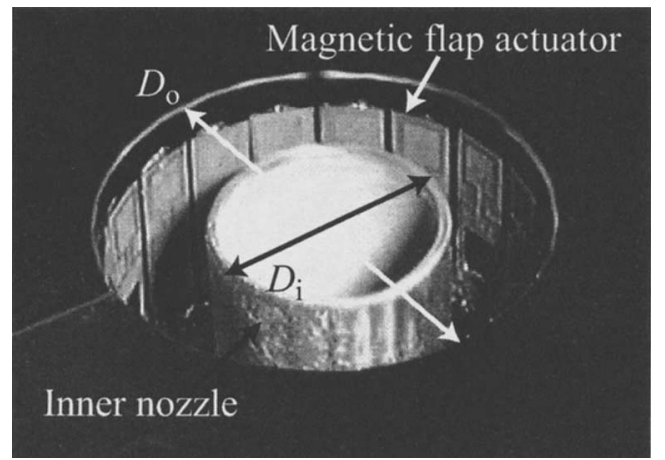

Fig. 8 Coaxial nozzle equipped with eighteen magnetic flap actuators (Kurimoto et al. ${ }^{(25)}$ )

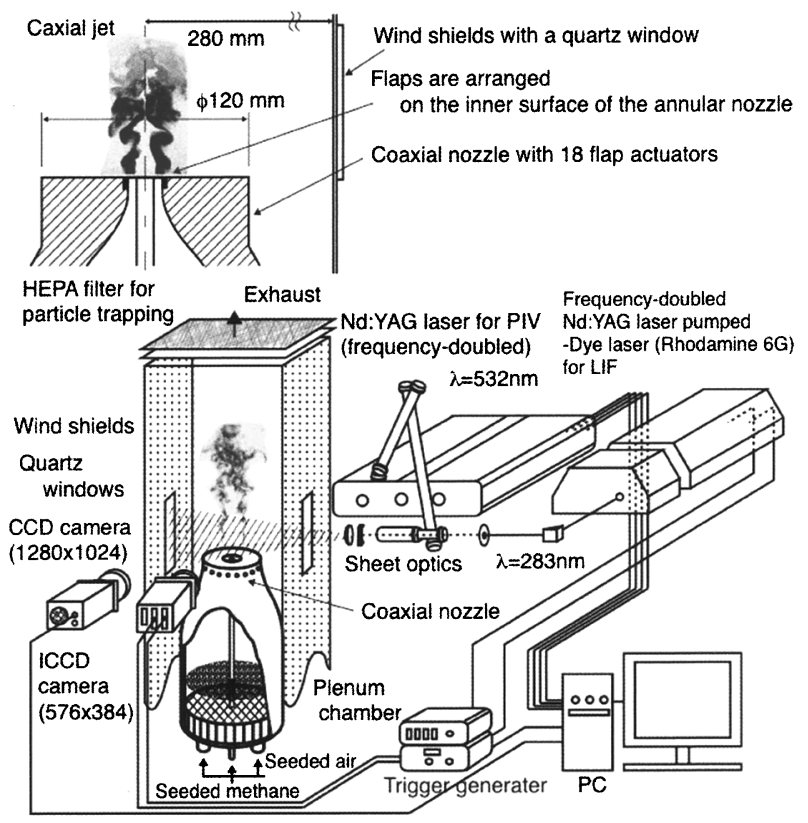

Fig. 9 Experimental setup for combustion control rates of the both streams are independently measured with mass flow meters. The coaxial jet is discharged vertically into the static ambient air, which is surrounded by four plates of windshields. Each plate has a quartz window, which provides optical access for a laser sheet and image acquisition. The test section is $1000 \mathrm{~mm}$ in height with a large square cross-section of $560 \times 560 \mathrm{~mm}^{2}$.

In representative results of the flame control described later, the bulk mean velocities of the inner and annular flows are respectively $U_{\mathrm{m}, \mathrm{i}}=1.2 \mathrm{~m} / \mathrm{s}$ and $U_{\mathrm{m}, \mathrm{o}}=$ $1.8 \mathrm{~m} / \mathrm{s}$, which corresponds to a $3.5 \mathrm{~kW}$ diffusion jet flame. The Reynolds number $\operatorname{Re}\left(=U_{\mathrm{m}, \mathrm{o}} D_{\mathrm{o}} / v_{\mathrm{o}}\right)$ is $2.4 \times 10^{3}$. The momentum flux ratio $m\left(=\rho_{\mathrm{o}} U_{\mathrm{m}, \mathrm{o}}^{2} / \rho_{\mathrm{i}} U_{\mathrm{m}, \mathrm{i}}^{2}\right)=4$, of which parameter is known to play a dominant role on the dynamics of coaxial jets (Favre-Marinet \& Camano Schettini ${ }^{(26)}$ ).

Instantaneous LIF images of the natural and controlled cold jets are shown in Fig. 10, where acetone vapor is separately seeded in the inner or outer flow. The inner and outer shear layers in the natural jet start to roll up into large-scale vortices at $z / D_{\mathrm{o}} \sim 2$ through the columnmode instability (Hussain \& Zaman ${ }^{(27)}$ ). The preferredmode frequency $f_{\mathrm{p}}$ of the vortex shedding is $57 \mathrm{~Hz}$, which corresponds to $S t_{\mathrm{p}}\left(=f_{\mathrm{p}} D_{\mathrm{o}} / U_{\mathrm{m}, \mathrm{o}}\right)=0.62$.

In the controlled jet with the saw-wave signal (Case 1), the outer shear layer is forced to roll up into large-scale vortices in phase with the flap motion, and the vortices pinch off the inner jet significantly (Fig. 10 (c) and (d)). Since the vortex is shed in phase with the flaps even if $f_{\mathrm{a}}$ is much smaller than $f_{\mathrm{p}}$, the vortex shedding is independent of the column-mode instability. In the present study, $f_{\mathrm{a}}$ is set to be $95 \mathrm{~Hz}\left(S t_{\mathrm{a}}=f_{\mathrm{a}} D_{\mathrm{o}} / U_{\mathrm{m}, \mathrm{o}}=1\right)$, which is the optimum frequency for flame stabilization. Figure 10 (e) and (f) shows the controlled jet with the square-wave signal at the same flapping frequency (Case 2). Unlike Case 1 , the distinct roll-up of the tracer is not observed in the outer shear layer.

Instantaneous images of the natural and controlled lifted flames are shown in Fig. 11. Smoke is introduced into the annular air flow, and the image is captured together with luminescence of the flame. As shown in Fig. 11 (a), the natural flame base is located near the end of the inner potential core, where large-scale vortices emerge intermittently. The lifted flame is unstable and easily blows off. This is related to the quasi-periodic passage of the strong vortices emerging at the potential core end. On the other hand, the controlled flames become stable. In Case 1, the large-scale vortices produced by the flaps are clearly observed at the flame base, which is anchored at $z / D_{\mathrm{o}} \sim 1.5$ (Fig. $11(\mathrm{~b})$ ). The total flame length was approximately $15 D_{0}$. Blue chemiluminescent emission was observed at the flame base with a luminous flame over a length of $\sim 12 D_{\mathrm{o}}$ downstream. These observations imply that partially premixed combustion is dominant at the flame base, while diffusion combustion prevails 


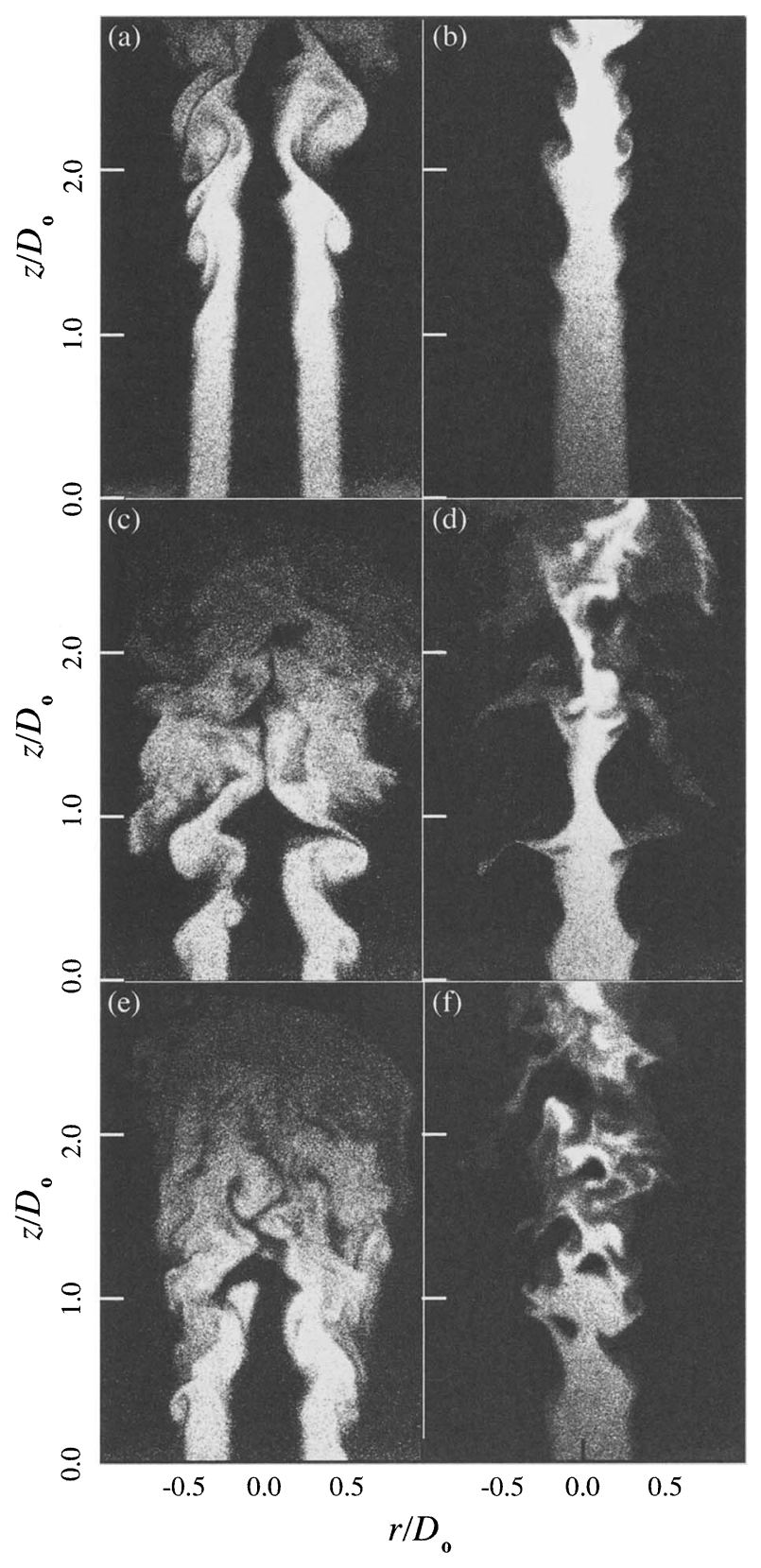

Fig. 10 Visualization of outer and inner jets. (a) \& (b) Natural, (c) \& (d) Case 1 (saw-wave signal, $S t_{\mathrm{a}}=f_{\mathrm{a}} D_{\mathrm{o}} / U_{\mathrm{m}, \mathrm{o}}=$ 1.0 ), (e) $\&$ (f) Case 2 (square-wave signal, $S t_{\mathrm{a}}=1.0$ )

downstream. In Case 2, the flame is held at $z / D_{\mathrm{o}} \sim 3.5$, which is further downstream of the inner potential core (Fig. 11 (c)). The total flame length was approximately $10 D_{\mathrm{o}}$ and blue chemiluminescent emission was observed at the flame base having a length of $\sim 6 D_{0}$. Therefore, partially premixed combustion should be dominant in the flame.

Figure 12 shows the maximum momentum flux ratio $m$ for sustaining a stable flame. A lifted flame is defined as stable when it is held at least for 3 minutes. In the natural flame, the blowoff limit is insensitive to $R e$, and it ranges from $m=1.5$ to 2 . On the other hand, in the controlled flames, it is significantly extended to larger $m$ for $R e>$

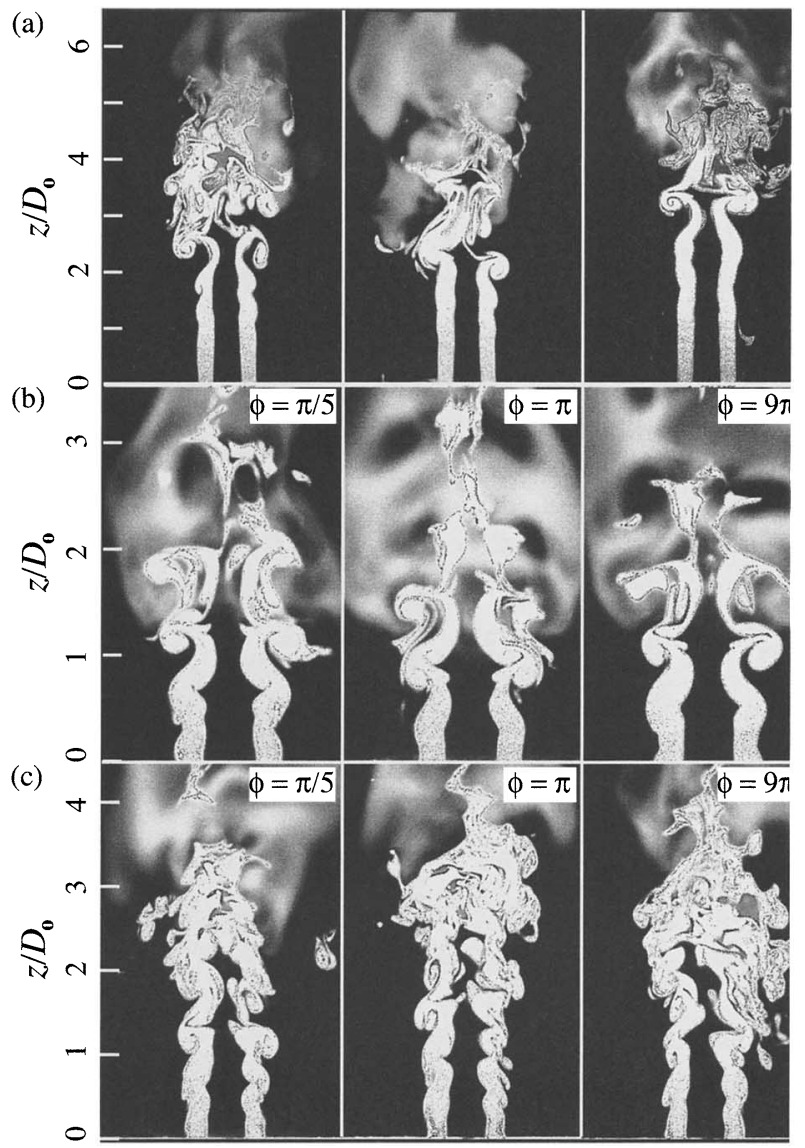

Fig. 11 Visualization of natural and controlled lifted flame with $S t_{\mathrm{a}}=1.0$. (a) Natural flame, (b) Case 1, (c) Case 2

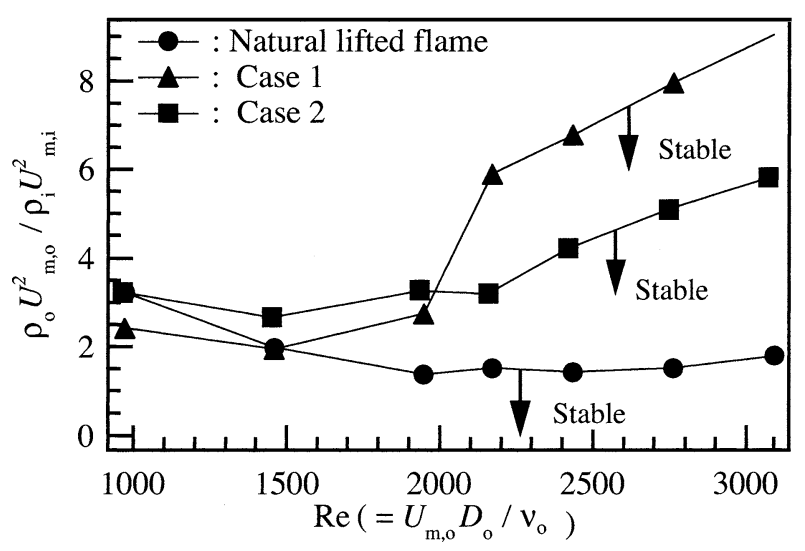

Fig. 12 Blowoff limits of natural and controlled flames

2000. The blowoff limit of Case 1 is approximately five times larger than that of the natural flame for $R e=2400$, whilst that of Case 2 is three times larger.

Many researchers have reported that a lifted flame is stabilized through excitation with piezoelectric actuators (Chao et al. ${ }^{(22)}$ ) or acoustic speaker (Chao et al. ${ }^{(28)}$; Baillot \& Demare $\left.{ }^{(29)}\right)$. They controlled lifted flames in the hysteresis region, where the exit flow velocity is so small that the natural flame base is held upstream of the jet potential core. The definition of stabilization in these studies 
is that the liftoff height becomes smaller by excitation. On the other hand, with the present control, the lifted flame is held stably even if the flow velocity is larger than the natural blowoff limit.

\section{DNS of Flow and Mixing Control in a Model Com- bustor}

We carried out direct numerical simulation (DNS) of scalar transport and mixing in a coaxial jet issued into a small model combustor (Mitsuishi et al. ${ }^{(30)}$ ). Analysis is made on the detailed mechanism of mixing enhancement achieved by an active control of the near-field largescale vortical structures. The main interest lies in the relationship between these vortical structures and associated scalar transport processes in the near field downstream of the intelligent nozzle exit.

Figure 13 shows the computational model used in this study. The flow domain is confined by lateral and bottom walls. The geometric conditions are $D_{\mathrm{o}} / D_{\mathrm{i}}=2$ and $D_{\mathrm{w}} / D_{\mathrm{o}}=2$ as the jet is now confined in a cylindrical space. The velocity ratio is $U_{\mathrm{m}, \mathrm{o}} / U_{\mathrm{m}, \mathrm{i}}=6.4$, which is close to the condition of a real gas turbine combustor. The incompressible continuity and Navier-Stokes equations and the transport equation of a passive scalar are used. The Reynolds number, $R e=U_{\mathrm{m}, \mathrm{o}} D_{\mathrm{o}} / v_{\mathrm{o}}=1320$, while that based on the downstream bulk mean velocity and $D_{\mathrm{w}}$ is 520. The Schmidt number, $S c$, of the passive scalar is assumed to be unity, which nearly corresponds to the diffusion of $\mathrm{CH}_{4}$ into air under the standard temperature and pressure. The governing equations are numerically solved by the finite difference method on the cylindrical coordinates (Fukagata \& Kasagi $\left.^{(31)}\right)$ with $(65,64$, $257)$ grid points in $(r, \theta, z)$ directions. In addition, we use the TVD scheme $\left(\operatorname{Harten}^{(32)}\right)$ for the advection term of the

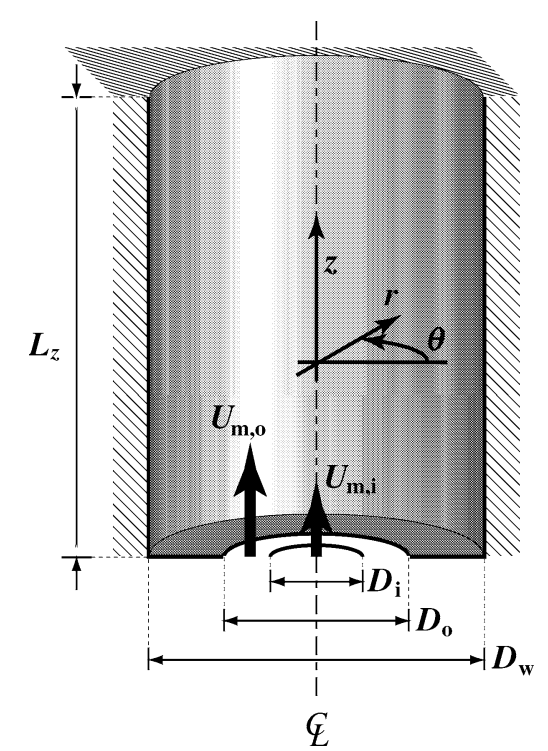

Fig. 13 Computational model of coaxial jet (Mitsuishi et al. ${ }^{(30)}$ ) scalar transport equation.

We focus on the axisymmetric mode of the intelligent nozzle, i.e., in-phase motion of all actuators, and model the motion of actuators as a change of an inlet velocity profile. The volumetric flow rate is kept constant and the outer nozzle radius is contracted sinusoidally as:

$$
R_{c t r l}(t)=R_{0}-\varepsilon\left(1-\cos \left(2 \pi S t_{\mathrm{a}} t\right)\right)
$$

In Fig. 14, instantaneous vortical structures and concentration of the scalar ejected from the central nozzle are visualized in the natural and controlled jets. The control amplitude and frequency are $\varepsilon=0.0125$ and $S t_{\mathrm{a}}=1$, at which the best mixing is observed in the experiment of Kurimoto et al. ${ }^{(25)}$ The vortical structures are identified by the second invariant of the deformation rate tensor. Without control, mixing between the central jet and the ambient fluid is very poor. A similar flow pattern is also observed in a confined annular jet at low Reynolds numbers (Sheen et al. $\left.{ }^{(33)}\right)$. With control, large-scale vortex rings are generated in the near field and they promptly break down intensifying scalar mixing, and this fact is in good agreement with the experiments described above.

The effects of control frequency on the mean scalar concentration along the central axis are shown in Fig. 15. Regardless of the frequency selected, better mixing is obtained than uncontrolled case. Various observations suggest that the control effects are classified into three regimes: (i) $S t_{\mathrm{a}}<0.7$, (ii) $0.7 \leq S t_{\mathrm{a}} \leq 1.1$, (iii) $1.1<S t_{\mathrm{a}}$. The regime of (ii) achieves the best mixing along the central axis among all tested cases. This is almost independent of $S t_{\mathrm{a}}$, so that the mixing enhancement is achieved by a certain mechanism which is not much affected by $S t_{\mathrm{a}}$.

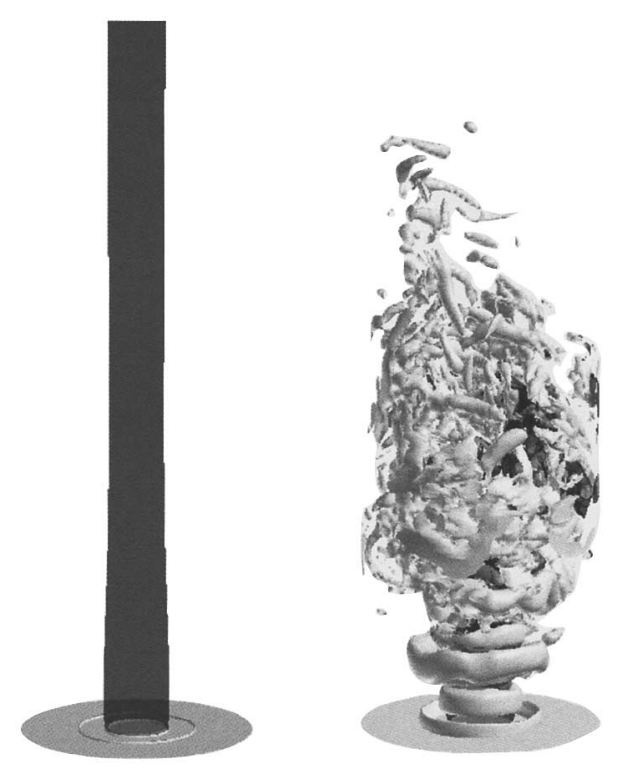

Fig. 14 Instantaneous vortical structures (white) and scalar concentration distributions (black). (a) natural jet, (b) controlled jet $\left(S t_{\mathrm{a}}=1, \varepsilon=0.0125\right)$ 


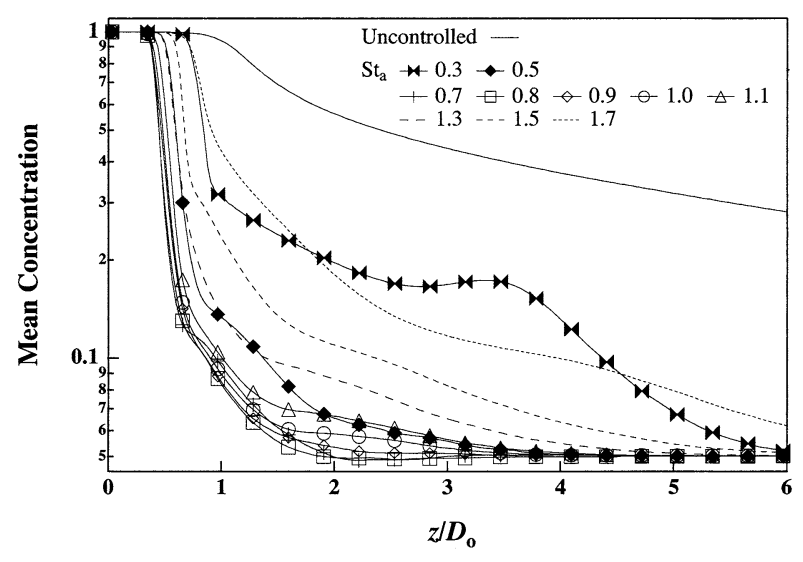

Fig. 15 Effect of control frequency on the mean scalar concentration along the central axis

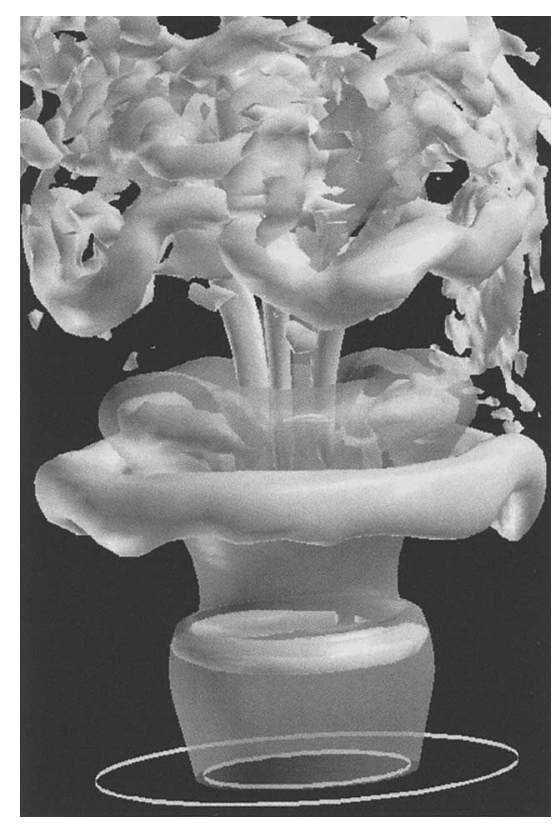

Fig. 16 Instantaneous vortical structures (white opaque) and scalar iso-surface (gray transparent)

Regime (i) is characterized by a restoration of the concentration downstream from the dilution around $z / D_{\mathrm{o}}<1$. The case of $S t_{\mathrm{a}}=0.3$ gives an especially clear hump at $z / D_{\mathrm{o}}=3.5$. In (iii), the mixing along the central axis is suppressed. Unlike (i), the central concentration decreases monotonically without any humps.

A close-up picture near the initial region is shown in Fig. 16. It clearly shows that the secondary streamwise vortices are generated in the inner shear layer, while the distortion of the vortex rings proceeds in the outer shear layer. The rib vortices thus formed play an important role to change the inner vortex rings from two- to threedimensional structures and consequent breakdown of the vortex rings. This three-dimensionalization of the inner vortex ring is closely related to the scalar transport. The overall picture obtained through the present series of DNS is represented in Fig. 17.

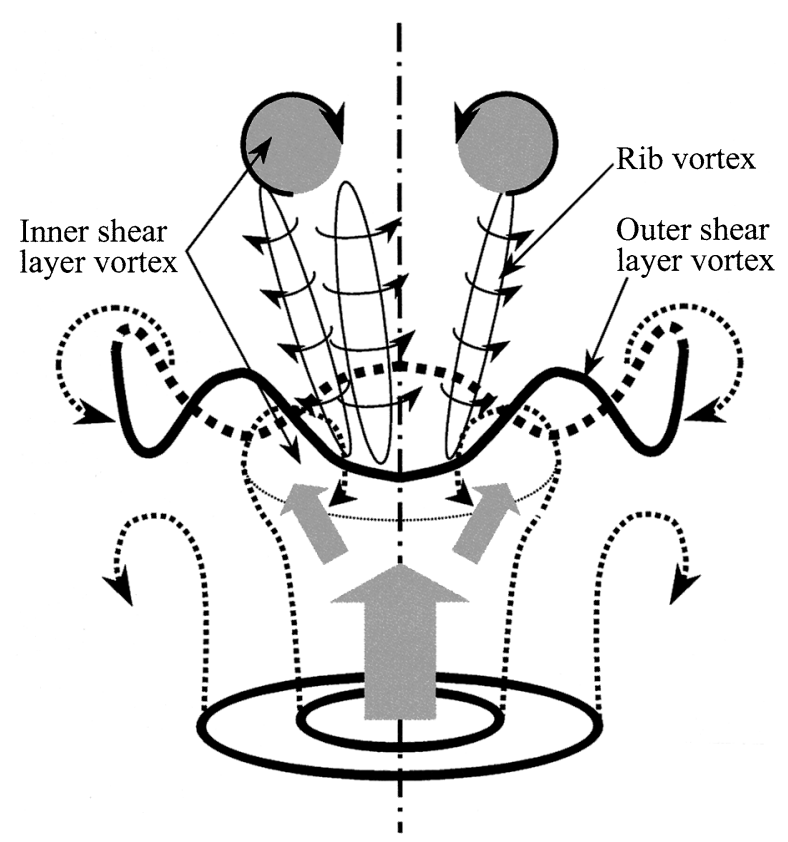

Fig. 17 Schematic of inner and outer shear layer development

\section{Concluding Remarks}

For the jet flow control, miniature electromagnetic flap actuators are developed and mounted on the periphery of an axisymmetric nozzle exit in order to induce various flow modes and enhance mixing processes. It is demonstrated that even weak disturbances introduced into the initial shear layer by these actuators can significantly modify the large-scale vortical structures. In particular, when flaps mounted on each half periphery of the jet nozzle are driven in anti-phase, the jet bifurcates into two branches without any bulk flow forcing. This control technique has been extended to the active control of methane/air mixing and diffusion combustion by using a coaxial jet nozzle with the same flap actuators. Dependent on the driving signal of flaps, the near-field vortical structures are significantly modified and two types of lifted flames having different stabilization mechanisms are achieved. As a result, the flame characteristics can be much improved in terms of stability (blowoff limit) and emission (NOx and UHC) even under lean combustion conditions.

Direct numerical simulation of the control of confined coaxial jet has also been carried out. Although the distributed actuators are modeled somewhat ideally, DNS clearly demonstrates enormous effects of the present control scheme on the initial shear layer dynamics and concentration mixing. It is found that distinct vortical structures appear in the inner and outer shear layer of the controlled coaxial jet. Namely, the secondary streamwise vortices (ribs) generated in the inner shear layer play an important role in the transition from two- to threedimensional structures and their subsequent breakdown, while the distortion of the primary vortex rings dominates 
the outer shear layer development.

Finally, despite the above substantial progress in active control of jet mixing and combustion, many issues still remain to be resolved for making the "smart control" feasible in future real applications. For the hardware, further downsizing of sensors/actuators, and development of actuators with low power consumption are required. In the software aspect, invention of control algorithms, particularly those with state feedback and learning, which can much effectively and flexibly control mixing, heat transfer, and chemical reaction, is essential.

\section{Acknowledgements}

The author deeply thanks Drs. H. Suzuki, Y. Suzuki, N. Kurimoto, K. Fukagata and Mr. A. Mitsuishi for their enormous contribution during the course of this work. The project was supported through the Grant-in-Aid for Scientific Research (No. 10355010) by the Ministry of Education, Culture, Sports, Science and Technology, and also through the research project on "Micro Gas Turbine/Fuel Cell Hybrid-Type Distributed Energy System" by the CREST of the Japan Science and Technology Corporation.

\section{References}

( 1 ) Kasagi, N., Kawaguchi, Y., Kodama, Y., Ogawa, S. and Yoshida, H., Progress in Smart Control of Turbulence, Proc. 6th Symp. Smart Control of Turbulence, Tokyo, (2005), pp.1-16.

( 2 ) Ho, C.M. and Tai, Y.C., Review: MEMS and Its Applications for Flow Control, ASME J. Fluids Eng., Vol.118 (1996), pp.437-447.

( 3 ) Huerre, P. and Monkewitz, P.A., Local and Global Instabilities in Spatially Developing Flows, Ann. Rev. Fluid Mech., Vol.22 (1990), pp.473-537.

( 4 ) Winant, C.D. and Browand, F.K., Vortex Pairing: the Mechanism of Mixing Layer Growth at Moderate Reynolds Number, J. Fluid Mech., Vol.63 (1974), pp.237-255.

( 5 ) Crow, S.C. and Champagne, F.H., Orderly Structure in Jet Turbulence, J. Fluid Mech., Vol.48 (1971), pp.547591.

( 6 ) Zaman, K.B.M.Q. and Hussain, A.K.M.F., Vortex Pairing in a Circular Jet under Controlled Excitation. Part 1. General Jet Response, J. Fluid Mech., Vol.101 (1980), pp.449-491.

( 7 ) Gutmark, E. and Ho, C.M., Preferred Modes and the Spreading Rates of Jets, Phys. Fluids, Vol.26 (1983), pp.2932-2938.

( 8 ) Mankbadi, R.R., Dynamics and Control of Coherent Structure in Turbulent Jets, Appl. Mech. Rev., Vol.45 (1992), pp.219-247.

( 9 ) Zaman, K.B.M.Q., Reeder, M.F. and Samimy, M., Control of an Axisymmetric Jet Using Vortex Generators, Phys. Fluids, Vol.6 (1994), pp.778-793.

(10) Reeder, M.F. and Samimy, M., The Evolution of a Jet with Vortex Generating Tabs: Real-Time Visualiza- tion and Quantitative Measurements, J. Fluid Mech., Vol.311 (1996), pp.73-118.

(11) Corke, T.C. and Kusek, S.M., Resonance in Axisymmetric Jets with Controlled Helical-Mode Input, J. Fluid Mech., Vol.249 (1993), pp.307-336.

(12) Smith, B.L. and Glezer, A., Vectoring and Small Scale Motions Effected in Free Shear Flows Using Synthetic Jet Actuators, AIAA-Paper 97-0213, (1997).

(13) Lee, M. and Reynolds, W.C., Bifurcating and Blooming Jets. Report TF-22, Thermosci. Div., Dept. Mech. Eng., Stanford University, (1985).

(14) Parekh, D.E., Leonard, A. and Reynolds, W.C., Bifurcating Jets at High Reynolds Numbers, Report TF-35, Thermosci. Div., Dept. Mech. Eng., Stanford University, (1988).

(15) Danaila, I. and Boersma, B.J., Direct Numerical Simulation of Bifurcating Jets, Phys. Fluids, Vol.12 (2000), pp.1255-1257.

(16) Pitts, W.M., Assessment of Theories for the Behavior and Blowout of Lifted Turbulent Jet Diffusion Flames, 22nd Symp. Combust., (1988), pp.809-816.

(17) Vanquikenborne, L. and Trigglen, A.V., The Stabilization Mechanism of Lifted Diffusion Flames, Combust. Flame, Vol.10 (1966), pp.59-69.

(18) Peters, N. and Williams, F.A., Liftoff Characteristics of Turbulent Jet Diffusion Flames, AIAA J., Vol.21 (1983), pp.423-429.

(19) Vervisch, L., Using Numerics to Help the Understanding of Non-Premixed Turbulent Flames, Proc. Combust. Inst., Vol.28 (2000), pp.11-24.

(20) Muniz, L. and Mungal, M.G., Instantaneous FlameStabilization Velocities in Lifted-Jet Diffusion Flames, Combust. Flame, Vol.111 (1997), pp.16-31.

(21) Fujimori, T., Riechelmann, D. and Sato, J., Effect of Liftoff on NOx Emission of Turbulent Jet Flame in High-Temperature Coflowing Air, 27th Symp. Combust., (1998), pp.1149-1155.

(22) Chao, Y.C., Huang, Y.W. and Wu, D.C., Feasibility of Controlling NOx Emissions from a Jet Flame by Acoustic Excitation, Combust. Sci. Tech., Vol.158 (2000), pp.461-484.

(23) Suzuki, H., Kasagi, N. and Suzuki, Y., Active Control of an Axisymmetric Jet with Distributed Electromagnetic Flap Actuators, Exp. Fluids, Vol.36 (2004), pp.498-509.

(24) Kurimoto, N., Suzuki, Y. and Kasagi, N., Coaxial Jet Control for Lifted Flame Stabilization with Arrayed Miniature Actuators, Proc. 12th Int. Symp. Appl. Laser Tech. Fluid Mech., Lisbon, CD-ROM Publication, Paper 30-2, (2004).

(25) Kurimoto, N., Suzuki, Y. and Kasagi, N., Active Control of Lifted Diffusion Flames with Arrayed Micro Actuators, Exp. Fluids, Vol.39 (2005), pp.995-1008.

(26) Favre-Marinet, M. and Camano Schettini, E.B., The Density Field of Coaxial Jets with Large Velocity Ratio and Large Density Differences, Int. J. Heat Mass Transfer, Vol.44 (2001), pp.1913-1924.

(27) Hussain, A.K.M.F. and Zaman, K.B.M.Q., The Preferred Mode of the Axisymmetric Mode, J. Fluid Mech., Vol.110 (1981), pp.39-71. 
(28) Chao, Y.C., Yuan, T. and Tseng, C.S., Effect of Flame Lifting and Acoustic Excitation on the Reduction of NOx Emissions, Combust. Sci. Tech., Vol.113 (1996), pp.49-65.

(29) Baillot, F. and Demare, D., Physical Mechanisms of a Lifted Nonpremixed Flame Stabilized in an Acoustic Field, Combust. Sci. Tech., Vol.174 (2002), pp.73-98.

(30) Mitsuishi, A., Fukagata, K. and Kasagi, N., On the Relationship between Large-Scale Vortical Structure and Scalar Transport Processes in a Controlled Confined Coaxial Jet, Proc. 4th Int. Symp. Turbulence \& Shear Flow Phenomena, Williamsburg, (2005), pp.841-846. (also, to appear in J. Turbulence, 2006)

(31) Fukagata, K. and Kasagi, N., Highly EnergyConservative Finite Difference Method for the Cylindrical Coordinate System, J. Comput. Phys., Vol.181 (2002), pp.478-498.

(32) Harten, A., On a Class of High Resolution TotalVariation-Stable Finite Difference Schemes, SIAM J. Num. Analys., Vol.21 (1984), pp.1-23.

(33) Sheen, H.J., Chen, W.J. and Wu, J.S., Flow Patterns for an Annular Flow over an Axisymmetric Sudden Expansion, J. Fluid Mech., Vol.350 (1997), pp.177-188. 